\title{
Téoros
}

Revue de recherche en tourisme

\section{L’Office des Congrès et du Tourisme du Grand Montréal : le défi des technologies de l'information}

\section{Pierre Bellerose et Stéphane Pipon}

Volume 14, numéro 3, automne 1995

Tourisme et technologies de l'information

URI : https://id.erudit.org/iderudit/1075081ar

DOI : https://doi.org/10.7202/1075081ar

Aller au sommaire du numéro

Éditeur(s)

Université du Québec à Montréal

ISSN

0712-8657 (imprimé)

1923-2705 (numérique)

Découvrir la revue

Citer cet article

Bellerose, P. \& Pipon, S. (1995). L’Office des Congrès et du Tourisme du Grand Montréal : le défi des technologies de l'information. Téoros, 14(3), 16-19.

https://doi.org/10.7202/1075081ar 


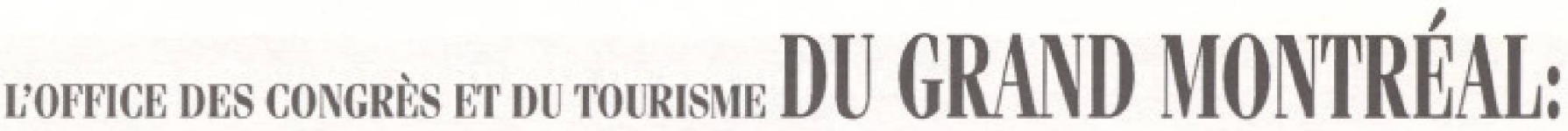 LE DÉFI DES TECHNOLOGIES
} DE L'INFORMATION

\author{
Pierre Bellerose et Stéphane Pipon
}

\author{
Pierre Bellerose \\ est directeur de la recherche, \\ du développement \\ et de l'accueil ainsi que \\ responsable des nouvelles \\ technologies à I'OCTGM. \\ Stéphane Pipon \\ est agent de recherche à la \\ Direction de la recherche et du \\ développement de l'OCTGM. \\ (e-mail: octgm@sun.cum.qc.ca) \\ (http://www,cum.qc.ca/octgm)
}

Depuls environ deux ans, les médlas se sont emparés d'un nouveau sujet à la mode pour l'appliquer à toutes les sauces et créer un raz-de-marée d'informatlons sans précédent dans l'histolre des technologles. En Janvler 1993, le terme "autoroute électronlque" est apparu 57 fols dans un Index de journaux et magazlnes de la presse anglophone. En Janvler 1994, le nombre dapparttlons a grimpé à 1480 fols! Malntenant, le nombre d'apparltions est tellement grand qu'on le subdlvise en une foule de rubriques différentes.

Cet engouement est llé, blen-sûr. aux transformations technologiques qul permettent, entre autres, un accès faclle à Internet. II ne faut pas se surprendre dans ce contexte de médlatisation accélérée de voir le grand public se tourner avec Intérêt vers les différents outllis composant les technologles de l'Information: Internet, CD-ROM, réseaux informatiques commerclaux (Compuserve, Amerlca On-line, Prodigy), systèmes de réservation, cartes à puce, etc.

Pour Internet seulement, le nombre d'usagers est phénoménal. On parle de plusleurs dizalne de millions d'Intervenants et d'une crolssance de $20 \%$ par mols.

Dans ce contexte, les principaux acteurs de chacun des secteurs d'actlvités économiques se positionnent face aux nouvelles technologles de l'informatlon et l'Industrle tourlstuque n'y échappe pas.

Le secteur tourlstique est d'autant plus Interpellé par la venue des technologles de l'Information qu'll subit lul-même les contrecoups d'Importantes transformatlions au seln des habitudes de consommations de toutes les cllentèles, mals spéciflquement celles du marché nord-américaln.

\section{Un nouveau touriste}

Selon plusleurs auteurs ${ }^{1}$. nous assistons actuellement à l'émergence d'un nouveau tourlste plus flexible, Indépendant et expérlmenté dont les besolns sont de plus en plus segmentés. Ce touriste cherche un prodult quul répond à ses asplrations, qu'll consldère souvent lul-même comme étant différentes de celles du vaste public. Mals en même temps, II souhalte un service raplde et de très grande qualité lul permettant de vlvre son expérience touristique quil répond à ses besolns spéciflques.
On observe donc l'apparition d'un nouveau groupe de touristes assez Important composé de personnes très scolarlsées et de voyageurs fréquents qui ne se retrouvent pas au seln du marché đle masse qul étalt la caractéristıque des années ' 70 et " 80 .

Ces personnes s'identuflent de plus en plus à un champ d'intérêt plutôt qu'à des caractéristques soclo-démographlques ou géographlques. Ce phénomène est déslgné sous le vocable de "créneaux" par les experts en marketing. Pour ces touristes, les campagnes publicltaires grand pubilc ont peu de prise. II faut falre un démarchage ciblé si on veut les attelndre. Dans ce contexte. le tradittonnel 4 " $\mathrm{S}$ " se défnnit maintenant autrement, solt : spéclallsation - segmentation sophisticatlon - satisfaction.

A un autre nlveau, les tendances générales nous montrent que les touristes font plus de voyages, mals d'une durée plus courte. Egalement. le temps entre la prise de décision et la date de départ est de plus en plus court. Pressé par le temps et plus scolarlsé, le nouveau tour'ste planlflera lul-même son voyage à parttr de son foyer. II aura donc besoln de plus en plus d'information dans des délals plus courts pour prendre une déclston éclairée. II voudra de plus une information de qualité́. mlse à four régullèrement.

C'est dans ce contexte mouvant que les intervenants touristlques s'intéressent fortement aux technologles de l'Informatlon.

\section{Vision et objectifs de l'office des Congrès et du Tourisme du Grand Montréal (OCTGM)}

LOCTGM est un organlsme prlvé à but non lucratuf fondé en 1919 quil a comme mandats principaux la promotion de Montréal à l'étranger et l'accuell des touristes. Pour arriver à ses fins, l'ofrice utilise depurs plusieurs décennles des outuls promotionnels et diaccueil traditionnels (encarts publicitaires, annonces, relations publiques, guldes touristiques, etc.).

Toutefols, devant l'utillsatlon élargle des technologles de l'Information, l'OCTGM se devalt de folndre les rangs des destlnatlons touristiques ayant une présence actlve dans ce secteur. C'est pourquol, à l'été 1994. le département de la recherche et du développement héritalt du nouveau mandat des technologles de l'informatlon.

\subsection{Les objectifs}

Au nlveau actuel du développement des technologles de l'Informatllon. Ies deux volets prloritalres sont l'accès à des bases de domnées en recherche et la diffusion d'Iuformattons sur la destunation Montréal. 
L'Infrastructure de communication actuelle ne permet pas encore d'actions promotionnelles à grand déplolement de même que de transaction directe du fournIsseur de services aux touristes en toute sécurité.

Dans ce contexte, les objectifs de l'OCTGM dans un horlzon de deux ans de notre présence dans les différents unlvers des technologles de l'Information consistent à:

1 Assurer un leadership de la destination Montréal et devenir l'organlsme de référence de diffusion de l'information tourlstique montréalalse sur les réseaux de dlstribution électronlque.

2 Maîtriser les différents outlls liés aux technologles de I'Information et en sulvre l'évolution (vellle technologique).

3 Donner une information complète et exacte aux cllents utllisant les technologles de l'information.

4 Assurer une présence convlvlale et de qualité.

5 Falre la mlse à jour régullère de l'information.

6 Établir un portralt-type de nos utullsateurs et analyser la répercussion de notre présence sur la fréquentation à Montréal

7 Favoriser et développer la présence des Intervenants tourlstıques de la réglon de Montréal au sein de ces nouveaux outlls technologiques

8 Rejolndre et convaincre un bassin de cllentèle composé de personnes scolarlsées et Intégrées dans une nouvelle culture technologique.

Pour atteindre ces objectlfs, prendre concrètement le virage technologique et assurer un leadershlp à l'OCTGM, nous avons priorisé deux projets moteurs. solt Internet et Travelflle.

\section{Internet}

\subsection{Le réseau}

Internet est un réseau de réseaux raccordant des dizaines de milliers d'organisations gouvernementales, d'institutions d'enselgnement, d'organisations sans but lucratif et de plus en plus d'organisations commerclales. Les usagers que l'on appelle "Internautes" accèdent à ce réseau en étant munis d'un ordinateur et d'un modem. Dans beaucoup de cas, les internautes accèderont à Internet en se branchant par l'intermédlalre de leur organtsation. Autrement, Ils se brancheront sur une base Individuelle chez un fournisseur de services Internet assurant l'accès à plusieurs individus au réseau.

La popularlté crolssante d'Internet depuls 2 ans s'expllque par l'apparition du World Wide Web (Web). Grâce à des loglclels que l'on appele "navigateurs", les Internautes navlguent virtuellement à travers Internet sur différents sites d'Informatlons avec une facllité déconcertante. Des llens hypertextes servent de "passerelle" entre les sites d'Informations. En cliquant sur différents poInteurs Identiflés par un soullgnement, les internautes obtjennent des pages d'Information agrémentées de textes, d'Images, de graphlques et même de vidéos. C'est la combinalson de ces modes de présentation et la facillté d'accès à l'information qul contribuent à la vague de popularité d'Internet.

D'Icl la fln 1995, certalns analystes avancent qu'll y aura près de $\mathbf{7 0}$ millions de personnes branchées à Internet. Une bonne part de ces personnes présentent un profil intéressant: pour l'Industrle tourlstıque. Plusieurs études rapportent que
I'Internaute possède un revenu élevé, qu'll est fortement scolarısé, qu'll voyage fréquemment et effectue des dépenses au-dessus de la moyenne lors de son voyage

\subsection{Le site Web de l'ocTGM}

Lé́tablissement d'un site Web sur Internet comporte un volet technique important. Lhébergement de l'information se falt sur le disque dur d'un serveur branché directement au réseau Internet. Le serveur est Identiflé par une adresse unlque par laquelle l'usager accèdera au sitte Web de l'organIsatıon. L'information trouvée par l'Internaute sulvra un mode organisationnel tenant compte de l'environnement dans lequel évolue l'organlsation gérant le site Web, des normes et critères de présentation sur le Web et flnalement des besolns et attentes de l'internaute.

Nous devons garder à l'esprit que la réallsation d'un site Web ne const.tue pas une fin en sol. Il faut s'assurer par la sulte que l'Information présentée à l'Intérleur du site ne devlenne pas périmée au point où les Internautes finlssent par consulter un site fantôme. La réallsation d'un site Web s'Inscrit dans un processus continu de mise à Jour de l"Information par le retralt ou l'ajout de données.

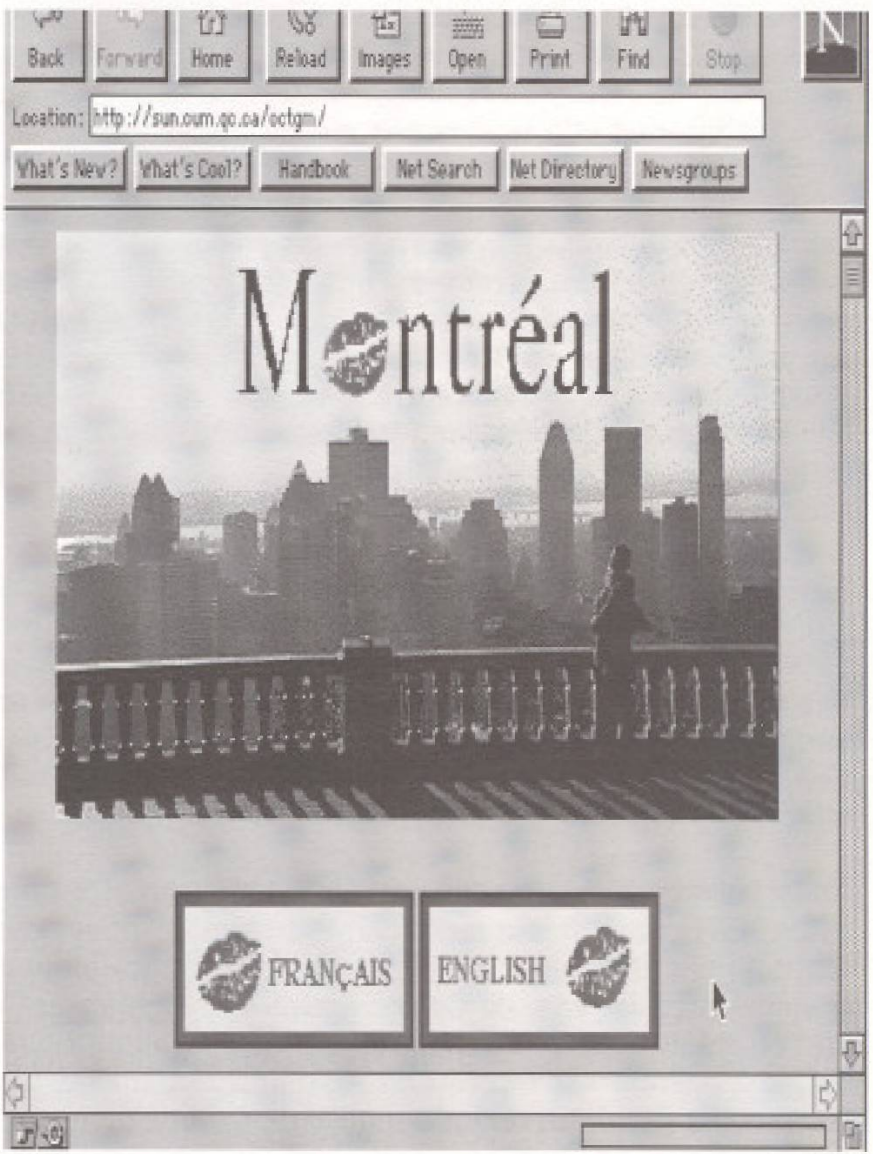

Dans le cas de roCTGM, tout le contenu informationnel est mis sur le serveur de la Communauté urbalne de Montréal (CUM). Les Internautes entrent sur le serveur en composant l'adresse du site Web: "http://www.cum.qc.ca/octgm/". Grâce à une entente de partenarlat entre les deux organlsations, la CUM apporte son expertise technlque tandis que l'OCTGM contribue par son expertise tourlstique. 
Au cours des six dernlers mols. l'ofnce s'est donc occupe à concevolr le site Web d'Informations touristiques le plus complet sur Montréal. Dans sa premlère phase de structuratIon, le site conçu en anglals et en françals présente une vingtalne de sections portant sur les differentes facettes du tourisme à Montréal.

Liobjectif premler de l'office est de devenlr le site Web́ montréalals de référence sur Internet. En attelgnant cet objectuf. l'offlce continue à assurer sa fonction de diffuseur d"luformations touristlques auprès des Individus et. des Intervenants touristiques interessés par notre destination.

A travers plus de 200 pages consutuées de textes, d'images et de cartes, les internautes obtiennent Instantanément en tout temps et de partout dans le monde toute I'Information dont lls peuvent avolr besoln pour la planiflcatlon de leur voyage. Dé|à, nous obtenons un nIveau d'achalandage attelgnant près de 1000 personnes par semalne qul sont majoritalrement anglophones.

\subsection{Le marketing}

Il est estlmé que 400 nouveaux sites d'Informations s'aJoutent présentement à chaque semalne au World Wide Web. Au Canada, on estume qu'll y aurait déjă plus de 1000 sites Web rellés au tourlsme sur Internet. Conséquemment à cette crolssance fulgurante du World Wide Web. I'OCTGM a donc adopté une démarche marketing pour faire connaître son site Web sur Internet et à travers son réseau traditionnel de distribution.

Sur Internet. à molns d'avolr l'adresse exacte d'un slte Web. II devlent difficlle de trouver l'Information désirée. Pour combler cette lacune, II exlste différents répertolres portant sur une panoplle de sujets afin de faciliter la recherche d'Informatlons. Ces répertoires regroupent par sujet des milliers de sites Web accessibles par l'établlssement de llens hypertextes à travers lesquels les Internautes pourront accéder au stte voulu.

Nous avons donc Identiflé différents répertolres reliés au tourisme dans lesquels l'Insertion d'un llen hypertexte avec notre site web a été réallsé. Conformément au plan marketing de l'OCTGM, nous avons sélectlonné ces répertolres sur une base géographlque et sectorlelle. Nous renforçons alnsl nos actlons traditionnelles portées sur ces différents marchés et assurons une présence étendue sur Internet.

Nous assumons également un rôle d'intermédialre entre notre site et ceux de nos membres. Par exemple, nous avons entre autres etabll des liens hypertextes avec les sites du Musée des beaux-arts, du Centre Canadlen d'Archltecture et du Festlval de Jazz. Nos actlons pour faire connaître la destlnation Montréal sur Internet font en sorte qu'll sera plus faclle pour ces intervenants d'obtenlr un nlveau d'achalandage élevé sur leurs pages Web. De leur côté, nos membres entreprennent également des actıons pour falre connaître leur site dans leur secteur tourlstique respect.lf ce qul en déflnitive falt connaître notre silte.

Plusleurs congrès possèdent leur propre site Web donnant toute l'Information utile aux congressistes dont de l'Informa-
Lon touristique. Notre démarche consiste icı à devenir le site offlclel d'Informations touristlques des congrès se tenant à Montréal en établissant des llens hypertextes mutuels.

Alnsi. en fournlssant un site complet d"Informations touristlques sur Montréal, nous pourrons contribuer à la réallsation de séfours pré et post congrès grâce à l'accès de nos pages Web à partir du site des différents congrès. Sous un autre angle, en énumérant dans nos pages les dirrérents sites Web des congrès se tenant à Montréal, nous permettrons aux congressistes connalssant la ville hôte de trouver facilement le site Web de leur assoclation. Nous pourrons ainsl contribuer positivement au ulveau de participation du congrès.

Notre démarche marketung Inclut aussi des actions sur notre réseau traditlonnel de distrlbutlon. Toutes nos campagnes publicitalres et promotlonnelles comportent les coordonnées de notre site Web. Nous utlliserons aussI notre papeterle (fax, lettres, cartes d'affalres, etc) pour promouvolr notre site. Nos publications tels que le gulde du planifloateur et le gulde tourlstique comportent également des indicatlons sur notre site.

Finalement, nous entreprenons des actlons à l'Interne auprès des employés. Des séances de famillarisation à Internet se sont tenues afın que les employés pulssent transmettre adéquatement de l'Information relative à notre site web. Le réseau de contact qu'lls ont développé à travers leur travall constitue une force pour promouvolr la destination Montréal vla Internet.

\section{TravelFile}

TravelFile est, une base de données informatısées fournlssant de I'Information sur différentes destinations touristiques à travers le monde. Conçue et gérée par la compagnle Applled Information Services (Whitefish. Montana, Etats-Unis). Travelfile constitue l'une des plus Importantes banques de données relative au tourlsme tant par son contenu que par les utIllsateurs qul peuvent y accéder.

Cette base de données est actuellement disponlble à travers 280000 termlnaux d'agents de voyages (vla les systèmes Informatıques de réservation des compagnles aérlennes tels que Sabre, Gallleo Internatlonal et Worldspan), 40000 organlsateurs d'événements et plus de 4 millons de consommateurs branchés à des réseaux commerclaux. Depuls peu. TravelFlle est également disponlble sur Internet aux milllons d'internautes.

Tous ces utIllsateurs ont accès à une base de données au sein de laquelle 120000 organismes et entreprises sont répertorlés. En 1994, le nombre de recherches effectuées par les usagers a attelnt 5 mllllons de recherches pour un total de 25 mllilons de pages-écran visualisées (une pageécran équivaut environ à un tlers d'une page $8.5 \times 11$ po).

\section{1 L'OCTGM sur TravelFile}

La démarche adoptée par l'office consistalt à fournir aux agents de voyage un site exhaustif d'Informations touristiques sur la destlnation Montréal tout en passant en revue l'ensemble de loffre montréalalse. II est à noter que les différentes pages-écran sont dépourvues d'images ou 
d'arrangements graphiques. La conception du slte s'est falte en respectant certalnes normes de présentatlon établles par TravelFile afln de malntenlr une untformité de présentation d'une destination à l'autre. Tout le traltement de l'Informatlon est falt à l'Interne afln d'assurer un contrôle total de l"Information diffusée par TravelFile sur leur système.

Pour accéder à l'information sur Montréal, les usagers de TravelFile passeront par la rubrique EZ Destination du menu princlpal de TravelFile. En Inscrivant. "Montreal pq". Ils arriveront directement au seul site d'Informations sur Montréal, celul de l'ocTGM. Le menu de la destlnation Montréal contlent près de quarante rubriques composées de textes et de données sur les entreprises et les événements.

\subsection{Les retombées}

Notre action sur TravelFile s'avère Intéressante dans la mesure où nous attelgnons un secteur touristique primordlal pour l'Industrle tourlstique, celul du réseau de distributıon. En ouvant leur ordinateur, les agents de voyages peuvent aller visiter notre site et ce, quand bon leur semble. Les systèmes Informatlques des compagnles aérlennes nous permettent donc d'attelndre un publlc cible pour alnsi dire captuf et par-dessus tout multiplicateur.

Depuls l'établlssement de notre site sur TravelFile el dale du 27 décembre 1994, nous avons obtenu une forte crolssance du niveau de consultation de nos pages-écrail. Par rapport au dernler trimestre de 1994 où l'information disponible sur Montréal provenalt de Tourisme Canada, nous avons obtenu une augmentation de $99 \%$ au deuxlème trimestre de 1995. Le nombre total de pages-écran vlsuallsées par les agents de voyages et les particullers s'est élevé à 70479 pour ce trimestre.

\section{Perspective}

Avec l'évolutlon rapide des technologles de l'information. l'OCTGM entend rester à l'affüt des différentes Innovatlons qui lul permettront de poursulvre ses efforts pour faire connaître le Montréal tourlstlque auprès des tourlstes et des multiplicateurs.

L'amélloration de la vitesse de transmission sur les réseaux de télécommunication. I'Instauration de centrales intégrées de réservatlons et d'informatlons par destlnatlon. lutilisatIon accrue du multımédia représentent quelques-unes des avenues de développement technologlque sur lesquelles nous devont porter une attention particullère. En général, les Intervenants touristiques sont d'avls que ces nouvelles technologles transformeront les différentes composantes de l'industrle tourlstique.

Dans un contexte d'internationalisation de la concurrence et de globallsation des marchés, II est essentiel d'adopter de nouveaux procédés par lesquels nous pourrons rejolndre un touriste de plus en plus élolgné. Par une approche proactive. nous devons être constamment en état de vellle technologique pour salsir les opportunltés qul nous permettront d'améllorer la performance de l'Industrle tourlstilque montrealalse.

\section{Bibliographie}

ARCHDALE, Gilbert, Computer reservation systems and public tourist offices. Tourlsm Management, févrler 1993. pp. $3-14$.

BECK, Nuala, La nouvelle économle, Montréal, Québec, Transcontlnales, $1994,232 \mathrm{p}$.

BROWN, Darin, An Internet Web site... what's it really cost, Strategy, aoot 7,1995, pp. 14-15.

BUHALIS, DImItrlos, RICIRMS as a strateglc tool for small and medium tourism enterprises, Tourism Management, octobre 1993, pp. 366-378.

CARROL, Jlm et BROADHEAD, Rlck. The Ganadlan Internet Advantage: Opportunities for Business and other organlsations, Scarborough, Canada, Prentice Hall Inc., 1995, 522 pages.

CARROL, JIII et BROADHEAD, RIck. The Canadlan Internet Handbook, Revised 1995 Edition. Scarborongh, Canada, Prentice Hall lnc., 1995, 911 pages.

DALLOZ, Xavler et PORTNOFF André-Yves, Les promesses de funlmédla. Futuribles, no. 191, octobre 1994, pp. 1136.

FLICHY, Patrice, Multimédla, objet-valise ou objet-frontlère, Futuribles, no. 191, octobre 1994, pp. 5-10.

POLLACK Ann. The Impact of information technology on desthatJon marketing. EIU Travel and Tourlsm Analyst. no. 3. 1995. pp. 66-83.

POON. Aullana. Tourism. Technology and competstive Strategles, Wallungord, UK, C.A.B. International, 1993. $370 \mathrm{p}$.

SCHMID, Beat. Electronic Markets in Tourlsm. The Tourlst Revlew, no. 2,1994, pp. 9-15.

SCHERTLER, Walter, Impact of New Information Technologies on Tourlsm Industry and Buslness. The Tourist Revlew, no. 2, 1994, pp, 2-8.

SOHIER, Dany J., Le gulde d'exploration de l'internaute, fonction avancée. Montréal, Québec, Les Éditions Loglques, 1995. 232 pages.

SOHIER. Dany J., Le gudde de survle de I'internaute, fonction de base, Montréal. Québec. Les Édltıons Loglques, 1994. 181 pages.

STIPANUK. David M. Tourism and techology. Interactlons and implications. Tourlsm Management. aoît 1993. pp. $267-278$

Notes

1 Voir cnte autres : Poon. Aulinna. Tourism, Technology and competitive strategles, C.A. A. Intertational. 1909. 